\title{
Alpha Error
}

National Cancer Institute

\section{Source}

National Cancer Institute. Alpha Error. NCI Thesaurus. Code C142387.

In statistics, alpha is the probability of a Type 1 error, when one incorrectly rejects the null hypothesis, creating a false positive. 